OPEN ACCESS

Edited by:

Yong Xia,

The Ohio State University,

United States

Reviewed by:

Osmar Antonio Centurion

National University of

Asunción, Paraguay

Tomi Petteri Laitinen,

Kuopio University Hospital, Finland

Amalia Peix,

Instituto de Cardiología y Cirugía

Cardiovascular, Cuba

*Correspondence:

Chunxia Qin

gin_chunxia@hust.edu.cn

Xiaoli Lan

xiaoli_lan@hust.edu.cn

Specialty section:

This article was submitted to

Cardiac Rhythmology

a section of the journal

Frontiers in Cardiovascular Medicine

Received: 02 June 2021 Accepted: 17 September 2021

Published: 15 October 2021

Citation:

Zheng D, Liu Y, Zhang L, Hu F, Tan X,

Jiang D, Zhou W, Lan $X$ and Qin C

(2021) Incremental Value of Left

Ventricular Mechanical Dyssynchrony

Assessment by Nitrogen-13 Ammonia

ECG-Gated PET in Patients With

Coronary Artery Disease.

Front. Cardiovasc. Med. 8:719565.

doi: 10.3389/fcvm.2021.719565

\section{Incremental Value of Left Ventricular Mechanical Dyssynchrony Assessment by Nitrogen-13 Ammonia ECG-Gated PET in Patients With Coronary Artery Disease}

\author{
Danzha Zheng 1,2, Yanyun Liu ${ }^{3}$, Lei Zhang ${ }^{1,2}$, Fan Hu ${ }^{1,2}$, Xubo Tan ${ }^{1,2}$, Dawei Jiang ${ }^{1,2}$, \\ Weihua Zhou ${ }^{4,5}$, Xiaoli Lan ${ }^{1,2 *}$ and Chunxia Qin ${ }^{1,2 *}$ \\ ${ }^{1}$ Department of Nuclear Medicine, Union Hospital, Tongji Medical College, Huazhong University of Science and Technology, \\ Wuhan, China, ${ }^{2}$ Hubei Key Laboratory of Molecular Imaging, Wuhan, China, ${ }^{3}$ Engineering Research Center of Molecular and \\ Neuro Imaging of Ministry of Education, School of Life Science and Technology, Xidian University, Xi'an, China, ${ }^{4}$ Department \\ of Applied Computing, Michigan Technological University, Houghton, MI, United States, ${ }^{5}$ Center of Biocomputing and Digital \\ Health, Institute of Computing and Cybersystems, and Health Research Institute, Michigan Technological University, \\ Houghton, MI, United States
}

Background: Phase analysis is a technique used to assess left ventricular mechanical dyssynchrony (LVMD) in nuclear myocardial imaging. Previous studies have found an association between LVMD and myocardial ischemia. We aim to assess the potential diagnostic value of LVMD in terms of myocardial viability, and ability to predict major adverse cardiac events (MACE), using Nitrogen-13 ammonia ECG-gated positron emission tomography (gPET).

Methods: Patients with coronary artery disease (CAD) who underwent Nitrogen-13 ammonia and Fluorine-18 FDG myocardial gPET were enrolled, and their gPET imaging data were retrospectively analyzed. Patients were followed up and major adverse cardiac events (MACE) were recorded. The Kruskal-Wallis test and Mann-Whitney $U$ test were performed to compare LVMD parameters among the groups. Binary logistic regression analysis, receiver operating characteristic (ROC) curve analysis, and multiple stepwise analysis curves were applied to identify the relationship between LVMD parameters and myocardial viability. Kaplan-Meier survival curves and the log-rank test were used to look for differences in the incidence of MACE.

Results: In total, 79 patients were enrolled and divided into three groups: Group 1 (patients with only viable myocardium, $n=7$ ), Group 2 (patients with more viable myocardium than scar, $n=33$ ), and Group 3 (patients with less viable myocardium than $\mathrm{scar}, n=39$ ). All LVMD parameters were significantly different among groups. The median values of systolic phase standard deviation (PSD), systolic phase histogram bandwidth (PHB), diastolic PSD, and diastolic PHB between Group 1 and Group 3, and Group 2 and Group 3 were significantly different. A diastolic PHB of $204.5^{\circ}$ was the best cut-off value to predict the presence of myocardial scar. In multiple stepwise analysis models, diastolic PSD, ischemic extent, and New York Heart Association (NYHA) classification were independent predictive factors of viable myocardium and myocardial scar. 
The incidence of MACE in patients with diastolic PHB > 204.5 was 25.0\%, higher than patients with diastolic PHB $<204.5^{\circ}$ (11.8\%), but the difference was not significant.

Conclusions: LVMD generated from Nitrogen-13 ammonia ECG-gated myocardial perfusion imaging had added diagnostic value for myocardial viability assessment in CAD patients. LVMD did not show a definite prognostic value.

Keywords: positron emission tomography, myocardial perfusion imaging (MPI), left ventricular mechanical dyssynchrony, coronary artery disease, myocardial viability

\section{INTRODUCTION}

Evaluation of myocardial viability is important in patients with coronary artery disease (CAD) when planning revascularization (1) and predicting the improvement of LV systolic function (2). The imaging methods to assess myocardial viability include coronary angiography, echocardiography, cardiac computed tomography (CT), cardiovascular magnetic resonance imaging, and nuclear myocardial imaging (1). Myocardial viability evaluated by myocardial perfusion imaging (MPI) and glucose metabolism imaging is regarded as the "gold standard" of non-invasive imaging (3). Compared with conventional imaging methods (4), myocardial perfusion and metabolism imaging assess regional myocardial physiology and function directly. However, the cost of both myocardial perfusion and metabolism positron emission tomography (PET) imaging is very high. The process to regulate blood glucose levels is complicated, especially in patients with diabetes (4). After timeconsuming preparation, the image quality of some patients is still not ideal. Therefore, it is necessary to look for an alternative method to distinguish viable myocardium from myocardial scar.

Phase analysis has emerged as an important technique to assess left ventricular mechanical dyssynchrony (LVMD) using nuclear imaging. Studies of the application of phase analysis have concentrated on the use of single-photon emission computed tomography (SPECT), in areas such as LVMD assessment in patients with left bundle branch block (5), optimizing patient selection for cardiac resynchronization therapy (CRT) (6), and improving CRT response $(7,8)$. Several studies have investigated the application of phase analysis for early diagnosis $(9,10)$, therapy (11), and prognosis evaluation (12, 13 ) in CAD patients. The worse degree of LVMD under stress perfusion imaging compared to images at rest was positively correlated with ischemic extent (14). Hibernating myocardium was an independent predictive factor of LVMD (15). So LVMD may have some relationship with ischemic myocardium and myocardial viability.

Limited studies have focused on gPET (16-18), especially using Nitrogen-13 ammonia ECG-gated MPI. PET can provide high-quality images with higher count rates, and increased spatial resolution compared with SPECT (4), and more positron tracers with ideal performance are available (19). The purpose of this study was to identify the diagnostic value of LVMD assessed with gPET MPI for myocardial viability assessment and its prognostic value in CAD patients.

\section{MATERIALS AND METHODS}

\section{Study Population}

This study was approved by the Institutional Ethical Committee of the Union Hospital, Tongji Medical College, Huazhong University of Science and Technology. Patients who underwent Nitrogen-13 ammonia gPET and Fluorine-18 FDG gPET for the myocardial viability assessment at the PET Center of Union Hospital from December 2015 to October 2019 were retrospectively enrolled. The inclusion criteria were adult patients with CAD as the main diagnosis confirmed by coronary angiography or coronary CT angiography. The exclusion criteria were: (1) severe valvular heart disease, (2) non-ischemic cardiomyopathy, and (3) severe arrhythmias such as left bundle branch block. Age, sex, history of myocardial infarction (MI) or old MI, risk factors for CAD, New York Heart Association (NYHA) functional classification were recorded. All the patients were followed up via medical records or telephone review to record the incidence of major adverse cardiac events (MACE), including unstable angina pectoris, myocardial infarction, heart failure, PCI, CABG, stroke, and cardiac death.

\section{Imaging Acquisition}

All patients underwent a rest Nitrogen-13 ammonia gPET and Fluorine-18 FDG gPET with a PET/CT scanner (GE Discovery VCT $^{\circledR}$, GE Healthcare, Milwaukee WI, USA) using a 1-day protocol. Aminophylline and caffeinated beverages were avoided for $48 \mathrm{~h}$ before the PET examination. All patients fasted for $6 \mathrm{~h}$. After connection of electrocardiographic (ECG) gating, and immediately after intravenous injection of $370-740 \mathrm{MBq}$ (10-20 mCi) Nitrogen-13 ammonia, rest Nitrogen-13 ammonia static PET images were acquired for $10 \mathrm{~min}$. Subsequently, oral glucose-loading with 25-50 g was performed, with supplemental insulin administered as needed (4). Between $1 \mathrm{~h}$ and $90 \mathrm{~min}$ after intravenous injection of $185-370 \mathrm{MBq}$ (5-10 mCi) Fluorine-18 FDG, PET data were acquired for $10 \mathrm{~min}$ under ECG gating. Images were generated using volume image protocol (VIP) replay with eight frames per R-to-R interval (4), screening out the frames with heart rates within the upper and lower $20 \%$. The images were reconstructed using the ordered subsets expectation maximization (OSEM) method.

\section{Imaging Analysis}

The data were input into commercial software (Emory Cardiac Toolbox@, ECTb, Atlanta GA, USA) (20) to measure left ventricular (LV) functional parameters, $L V$ wall thickening score, LVMD parameters, ischemic extent, and myocardial viability, including the degree of viable myocardium and myocardial scar. 


\section{Myocardial Viability Assessment and Grouping}

The ischemic extent was assessed on Nitrogen-13 ammonia MPI, obtained from ECTb software by using the default threshold of $50 \%$ (20). The algorithm of ECTb software searches for the maximal counts in the entire LV myocardial distribution and identifies those myocardial segments that fall below 50\% of this maximal value as ischemic, using the extensively validated method of O'Connor et al. (21). Ischemic extent was defined as the ratio of ischemia area to the whole LV area. The areas with normal FDG uptake were defined by comparing with the normal database provided by software developer, and the FDG uptake was scaled to equate its average value in the normal region with that in the perfusion study. The FDG tool differentiate viable myocardium and myocardial scar by a threshold of $40 \%$ of the maximum FDG counts in LV myocardium. In the area with FDG distribution above the threshold coexistent with relatively decreased perfusion was considered viable myocardium (perfusion/metabolic mismatch), under the threshold coexistent with decreased perfusion was considered as myocardial scar (perfusion/metabolic match) $(4,20)$. The degree of viable myocardium/myocardial scar was defined as the ratio of viable myocardium area/scar area to the whole LV area. CAD Patients were grouped by comparison of their own degree of viable myocardium and myocardial scar. Group 1 included patients with viable myocardium only. Group 2 included patients with more viable myocardium than their own myocardial scar. Group 3 included patients with more myocardial scar than their own viable myocardium.

\section{LVMD Analysis}

The software obtained the change of myocardial wall thickness by the change in maximum counts at the same myocardial region. After matching the onset time when the myocardial region starts contracting, the time distribution of myocardial contraction percentage in the cardiac cycle was obtained (22). The phase histogram was used to display the correlation of phase and the percentage of myocardial contraction, with the $x$ axis representing the phase (time), and the $y$-axis representing the percentage of myocardium that starts contracting at the corresponding time on the $x$-axis. Phase standard deviation (PSD) and phase histogram bandwidth (PHB) were the most common parameters to describe systolic LVMD, as the standard deviation and 95\% width of phase distribution, respectively (22). Similar methods were used to assess LVMD at LV diastole. The LV wall thickening curve was approximated by the 3-harmonic function for diastolic LVMD after undergoing a count drop

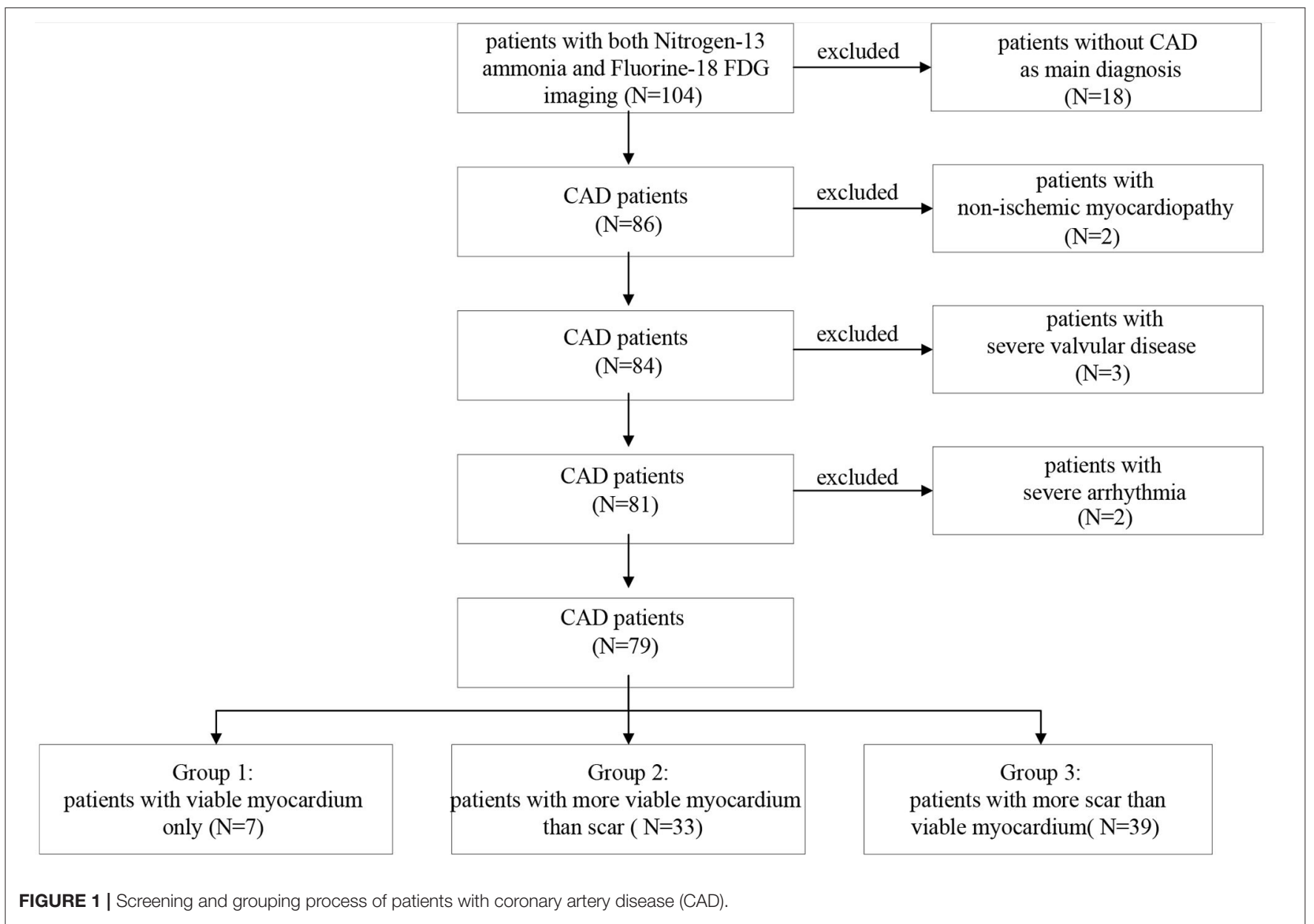


correction. Use diastolic PSD and PHB to describe diastolic LVMD (23).

\section{Statistical Analysis}

Data were processed by commercial statistical software (IBM SPSS Statistics 25.0, IBM, Armonk NY, USA). Normally distributed continuous variables are expressed as mean \pm standard deviation, non-normally distributed continuous variables as median and interquartile range (25th, 75th), and categorical variables as numbers or percentages. Either analysis of variance or the Kruskal-Wallis test was used to compare LV functional and LVMD parameters among groups. MannWhitney $U$ test was performed for pairwise comparisons. Binary logistic and multiple stepwise regression analyses were performed to screen for parameters predictive of myocardial viability. A receiver operating characteristic (ROC) curve was generated. The cut-off value was determined by the Youden index. Kaplan-Meier survival curve and log-rank test were generated to assess for differences in the incidence of MACE. $P$ $<0.05$ was considered statistically significant.

\section{RESULTS}

\section{Patient Characteristics}

A total of 104 patients underwent both Nitrogen-13 ammonia and Fluorine-18 FDG myocardial gPET imaging; 86 patients were diagnosed with CAD. After excluding two cases of nonischemic cardiomyopathy, three cases of severe valvular disease, and two cases with severe arrhythmia, a total of 79 patients were included in this study (Figure 1). Age, sex, MI or old MI, the proportion of overweight and obese, risk factors for CAD, LV functional parameters, LVMD parameters, wall thickening scores, and myocardial viability assessments of all patients are shown in Table 1. NYHA functional classification III and IV were the most common functional classification in patients. In addition, LV systolic dysfunction and regional wall motion abnormality commonly existed in the study population.

\section{Comparison of LV Functional and LVMD Parameters Among Groups}

There were 7 patients in Group 1, 33 in Group 2, and 39 in Group 3 (Figure 1). The number of patients with MI was $6(85.7 \%)$, $14(42.4 \%)$, and $25(64.1 \%)$ in each group, respectively. Among them, 2 (28.6\%) in Group 1, 9 (27.3\%) in Group 2 and 19 (48.7\%) in Group 3 had old MI. In the comparison of LV functional parameters, there were no significant differences among the three groups in LV ejection fraction (LVEF), end-diastolic volume (EDV), end-systolic volume (ESV), peak filling rate (PFR), or time of peak filling rate (TPFR) (Table 2).

The comparisons of LVMD parameters, including systolic PSD, systolic PHB, diastolic PSD, and diastolic PHB among groups were shown in Figure 2. All the LVMD parameters were statistically different among groups, the $P$-value of systolic PSD, systolic PHB, diastolic PSD, and diastolic PHB was 0.007 , $0.001,0.001$, and 0.001 , respectively. In pairwise comparison after Bonferroni's correction, the median values were significantly different between Group 1 and Group 3, $P=0.044$, 0.017, 0.019,
TABLE 1 | Basic characteristics of the CAD patients [proportion, mean \pm SD or median (25th, 75th)].

\begin{tabular}{|c|c|}
\hline & CAD patients $(N=79)$ \\
\hline Age (y) & $56.8 \pm 9.9$ \\
\hline Male & $68 / 79(86.1 \%)$ \\
\hline Overweight $(25<=\mathrm{BMl}<30)$ & $27 / 79(34.2 \%)$ \\
\hline Obese (BMl > 30) & $6 / 79(7.6 \%)$ \\
\hline Ml or Old Ml & $45 / 79(57.0 \%)$ \\
\hline \multicolumn{2}{|l|}{ CARDIAC RISK FACTORS OF CAD } \\
\hline Family history of cardiovascular disease & $15 / 79(19.0 \%)$ \\
\hline Smoke & $45 / 79(57.0 \%)$ \\
\hline Hyperlipemia & $19 / 79(24.1 \%)$ \\
\hline Hypertension & $40 / 79(50.6 \%)$ \\
\hline Diabetes & $31 / 79(39.2 \%)$ \\
\hline \multicolumn{2}{|l|}{ NYHA FUNCTIONAL CLASSIFICATION } \\
\hline ॥ & 15/79 (19.0\%) \\
\hline III & $41 / 79(51.9 \%)$ \\
\hline IV & 23/79 (29.1\%) \\
\hline \multicolumn{2}{|c|}{ LEFT VENTRICULAR FUNCTIONAL PARAMETERS } \\
\hline EF (\%) & $26.0(19.0,36.0)$ \\
\hline $\mathrm{EDV}(\mathrm{ml})$ & $242.0(180.0,334.0)$ \\
\hline ESV (ml) & $178.0(118.0,251.0)$ \\
\hline PFR (EDV/RR) & $1.2(0.9,1.6)$ \\
\hline TPFR (\%RR) & $23.2(15.1,38.2)$ \\
\hline \multicolumn{2}{|c|}{ REGIONAL WALL MOTION PARAMETERS } \\
\hline Systolic PSD $\left(^{\circ}\right)$ & $77.1(62.2,85.2)$ \\
\hline Systolic PHB $\left(^{\circ}\right)$ & $252.0(201.0,283.0)$ \\
\hline Diastolic PSD $\left(^{\circ}\right)$ & $81.3(66.9,90.0)$ \\
\hline Diastolic PHB $\left(^{\circ}\right)$ & $268.0(213.0,295.0)$ \\
\hline Wall thickening score (\%) & $11.3 \pm 7.3$ \\
\hline \multicolumn{2}{|l|}{ MYOCARDIAL VIABILITY PARAMETERS } \\
\hline Ischemic extent (\%) & $25.8 \pm 11.5$ \\
\hline Viable myocardium (\%) & $11.2(5.4,20.4)$ \\
\hline Myocardial scar (\%) & $7.6(2.3,22.8)$ \\
\hline
\end{tabular}

$C A D$, coronary artery disease; $S D$, standard deviation; $P S D$, phase standard deviation; $P H B$, phase histogram bandwidth; EF, ejection fraction; EDV, end-diastolic volume; ESV end-systolic volume; PFR, peak filling rate; TPFR, time of peak filling rate; RR, RR interval.

and 0.012 for systolic PSD, systolic PHB, diastolic PSD, and diastolic PHB, respectively. They were also significantly different between Group 2 and Group $3(P=0.030,0.007,0.005$, and 0.010 , respectively). No significant difference was found between Group 1 and Group 2. Examples of LVMD analysis were shown in Figure 3.

\section{Binary Logistic Regression Analysis and ROC Curves for Myocardial Viability}

Age, sex, BMI, cardiac risk factors, NYHA functional classification, LV functional parameters, ischemic extent, wall thickening score, and LVMD parameters were included in the binary logistic regression model for predicting the presence of myocardial scar. Diastolic PHB was the only factor to identify myocardial scar, Odds ratio (95\% Confidence Interval) $=1.015$ (1.005-1.025), $P=0.004$. The cut-off value for diastolic PHB to 
discriminate between patients with and without myocardial scar was $204.5^{\circ}$, the Youden index $=0.548$ (Figure 4). The sensitivity and specificity were 83.3 and $71.4 \%$, respectively. The area under the ROC curve was $0.751, P=0.029$.

\section{Multiple Stepwise Regression Analysis for the Prediction of the Myocardial Viability}

Age, sex, BMI, cardiac risk factors, NYHA functional classification, LV functional parameters, ischemic extent,

TABLE 2 | Comparison of left ventricular functional parameters among groups [median (25th,75th)].

\begin{tabular}{lccccc}
\hline & Group 1 & Group 2 & Group 3 & H & P-value \\
\hline EF (\%) & 29.0 & 26.0 & 24.0 & 3.047 & 0.218 \\
& $(23.0,65.0)$ & $(19.0,37.0)$ & $(19.0,34.0)$ & & \\
EDV $(\mathrm{ml})$ & 207.0 & 242.0 & 247.0 & 1.808 & 0.405 \\
& $(87.0,341.0)$ & $(162.0,363.0)$ & $(196.0,283.0)$ & & \\
ESV $(\mathrm{ml})$ & 146.0 & 187.0 & 193.0 & 2.001 & 0.368 \\
& $(29.0,262.0)$ & $(114.5,289.0)$ & $(141.0,229.0)$ & & \\
PFR & 1.9 & 1.1 & 1.2 & 2.404 & 0.301 \\
(EDV/RR) & $(1.1,2.7)$ & $(0.9,1.4)$ & $(0.9,1.6)$ & & \\
TPFR & 22.5 & 24.8 & 21.3 & 0.349 & 0.840 \\
$(\% R R)$ & $(16.6,28.7)$ & $(15.3,40.5)$ & $(14.5,38.1)$ & &
\end{tabular}

Group 1, patients with viable myocardium only; Group 2, patients with more viable myocardium than scar; Group 3, patients with more scar than viable myocardium. EF, ejection fraction; EDV, end-diastolic volume; ESV, end-systolic volume; PFR, peak filling rate; TPFR, time of peak filling rate. wall thickening score, and LVMD parameters were included in the multiple regression stepwise model to predict viable myocardium and myocardial scar. Ischemic extent, diastolic PSD, and NYHA functional classification were identified as the independent factors to predict viable myocardium (coefficient of determination $\left.\left[R^{2}\right]=0.329\right)$ and myocardial scar $\left(R^{2}=0.373\right)$, as shown in Table 3 .

\section{Prognostic Value of LVMD}

The mean follow-up time was $17.9 \pm 12.9$ months. Of the 79 patients, 65 had follow-up results, in which MACE occurred in 14 (17.7\%), 44 did not have MACE, seven died of unknown causes. Kaplan-Meier survival curves of patients with diastolic PHB > $204.5^{\circ}$ and $\mathrm{PHB}<204.5^{\circ}$ were shown in Figure 5. The incidence of MACE in patients with diastolic $\mathrm{PHB}<204.5^{\circ}(11.8 \%)$ was lower than that in patients with diastolic PHB $>204.5^{\circ}(25.0 \%)$. However, there was no statistical difference in the incidence of MACE by log-rank test $(P=0.340)$.

\section{DISCUSSION}

This study focused on whether LVMD contributed to the diagnosis of viable myocardium and myocardial scar by Nitrogen-13 ammonia ECG-gated MPI. LVMD parameters differed among groups, which suggests the usefulness of LVMD to distinguish patients with different proportions of myocardial scar, which confirmed our hypothesis. Diastolic PHB contributed to predicting the presence of myocardial scar by the binary logistic regression model, and the cut-off value was $204.5^{\circ}$.

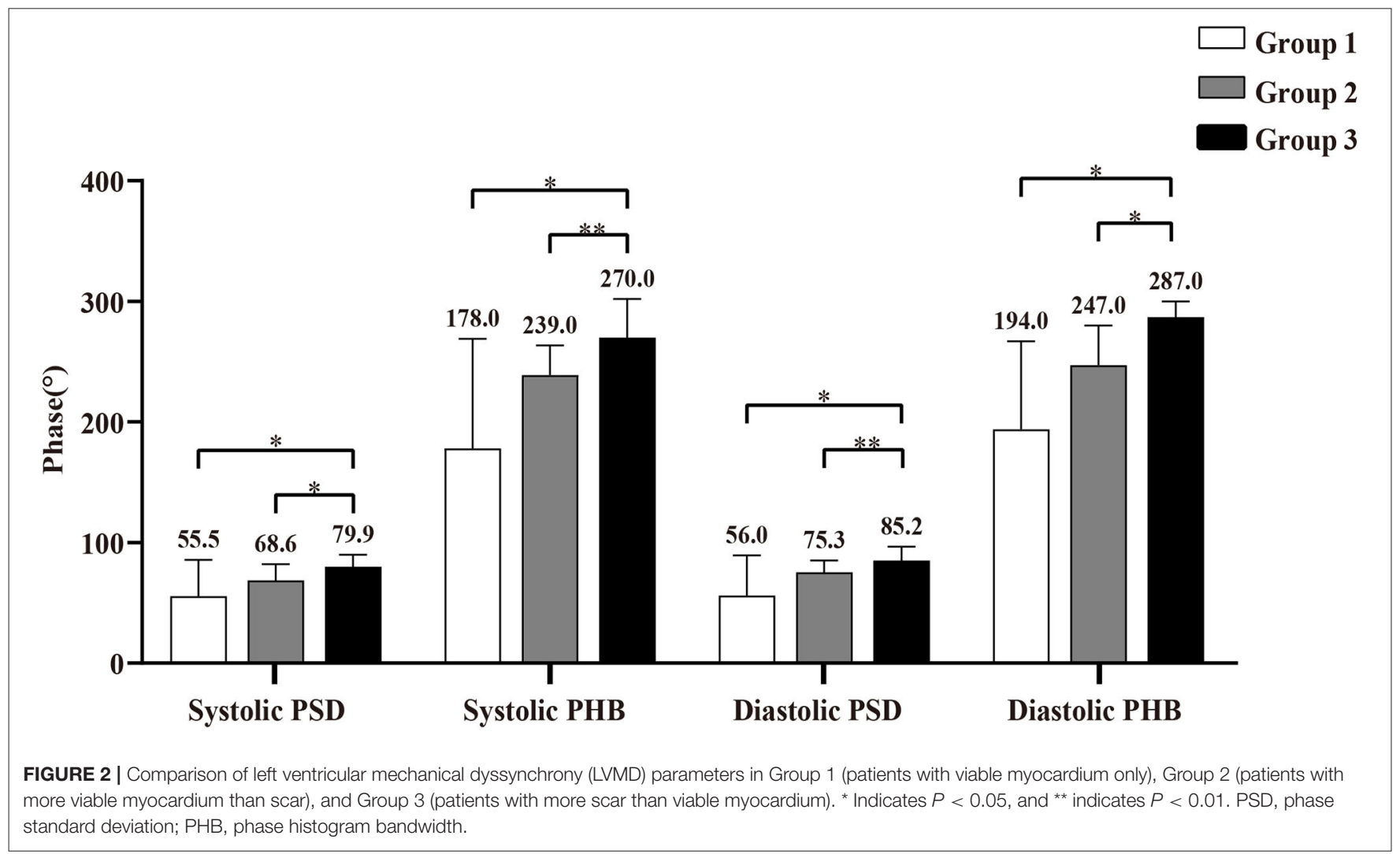




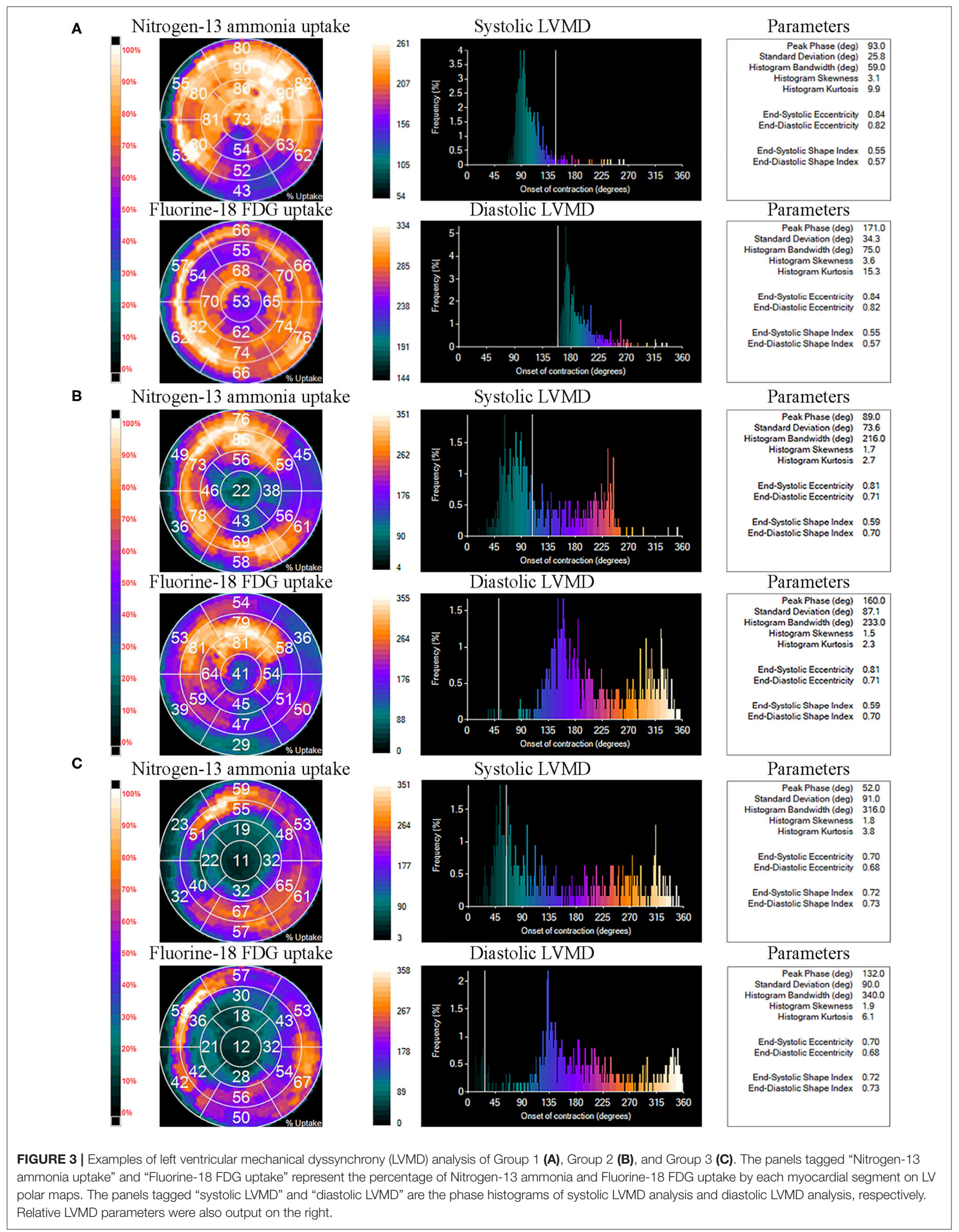




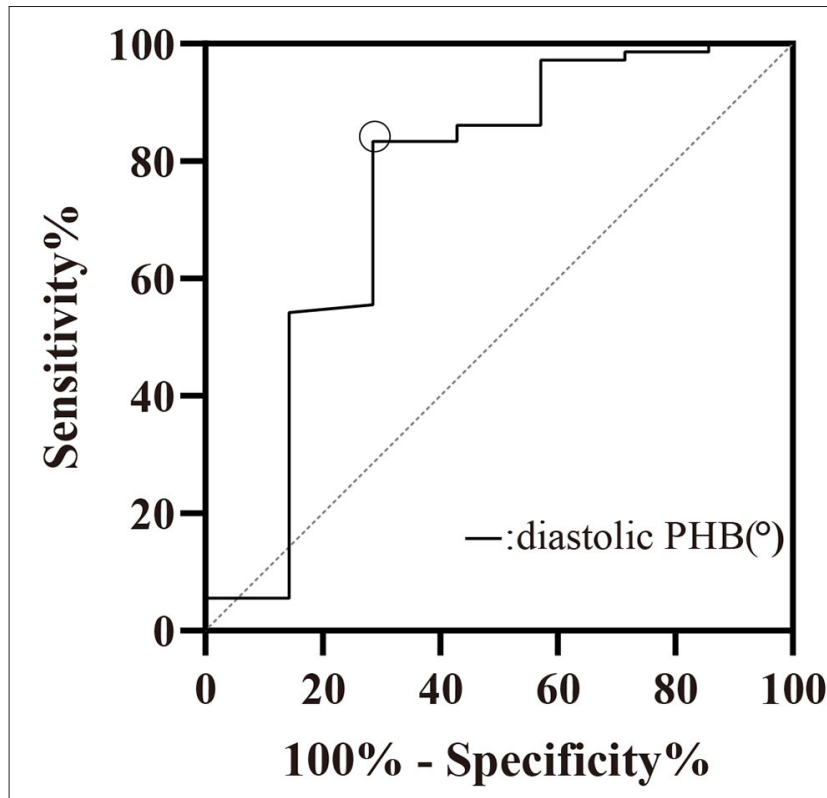

FIGURE 4 | ROC curve of diastolic $\mathrm{PHB}\left(^{\circ}\right)$ for the prediction of myocardial scar. Cut-off value: diastolic PHB $>204.5^{\circ}$, Sensitivity $=83.33 \%$, Specificity $=71.43 \%$. PHB, phase histogram bandwidth.

TABLE 3 | The multiple stepwise regression analysis for the prediction of the myocardial viability.

\begin{tabular}{|c|c|c|c|c|c|}
\hline $\begin{array}{l}\text { Dependent } \\
\text { variable }\end{array}$ & $\begin{array}{l}\text { Independent } \\
\text { variable }\end{array}$ & B & $\beta$ & Significance & Adjusted $R^{2}$ \\
\hline \multirow[t]{3}{*}{$\begin{array}{l}\text { Viable } \\
\text { myocardium (\%) }\end{array}$} & $\begin{array}{l}\text { Ischemic } \\
\text { extent (\%) }\end{array}$ & 0.006 & 0.652 & $P<0.001$ & 0.329 \\
\hline & Diastolic PSD $\left({ }^{\circ}\right)$ & -0.002 & -0.315 & $P<0.01$ & \\
\hline & NYHA & 0.033 & 0.209 & $P<0.05$ & \\
\hline \multirow[t]{3}{*}{$\begin{array}{l}\text { Myocardial scar } \\
(\%)\end{array}$} & $\begin{array}{l}\text { Ischemic } \\
\text { extent (\%) }\end{array}$ & 0.004 & 0.392 & $P<0.001$ & 0.373 \\
\hline & Diastolic PSD $\left(^{\circ}\right)$ & 0.002 & 0.304 & $P<0.01$ & \\
\hline & NYHA & -0.033 & -0.202 & $P<0.05$ & \\
\hline
\end{tabular}

NYHA, New York Heart Association functional classification; B, partial regression coefficient; $\beta$, standard regression coefficient.

Diastolic PSD, ischemic extent, and NYHA classification were identified in a multiple regression stepwise model to predict the degree of viable myocardium and myocardial scar. LVMD from phase analysis of Nitrogen-13 ammonia ECG-gated MPI was likely beneficial to the diagnosis of myocardial viability in CAD patients with LV systolic dysfunction. However, the value of LVMD as a predictor of MACE of CAD patients still needs further study.

The application of LVMD for CAD diagnosis was focused on the relationship between LVMD with coronary artery stenosis and myocardial perfusion defects in previous studies (9, 10, 24, 25). LVMD has been suggested to be associated with occult atherosclerosis in patients with normal coronary angiography but with reversible perfusion defects (9) and subclinical atherosclerosis (24). Moreover, the correlation between LVMD and myocardial perfusion defects assessed by SPECT overwhelmed the diagnosis of CAD by coronary angiography in patients with anginal chest pain and known or suspected CAD (10). Early detection and treatment of LVMD may slow the appearance of cardiac dysfunction in patients with ischemic heart disease (25). However, there is a paucity of studies linking myocardial viability and LVMD. It has been suggested that hibernating myocardium is an independent predictive factor of LVMD (15); thus, we hypothesized that LVMD may contribute to distinguishing the relative proportion of viable myocardium and myocardial scar by Nitrogen-13 ammonia gated MPI alone. Our results confirmed this hypothesis.

All the LVMD parameter values were larger in patients with a higher proportion of myocardial scar (Group 3) than in patients with a lower proportion (Group 2) and without myocardial scar (Group 1). A diastolic PHB cut-off of $204.5^{\circ}$ predicted the presence of myocardial scar. The results suggested that combining LVMD with ischemic extent may provide clinicians with an intuitive impression on which patient has more proportion of viable myocardium. Previously, it was difficult to have a view of the degree of myocardial scar and viable myocardium on rest MPI alone. In the multiple stepwise regression analysis for the prediction of myocardial viability, we noticed the degree of myocardial scar increased and viable myocardium decreased with increasing diastolic PSD, indicating that diastolic PSD has diagnostic value in terms of myocardial viability in Nitrogen-13 ammonia ECG-gated MPI. There are several possible explanations. First, myocardial scar is one of the main features in the progression of CAD. It decreases regional myocardial electromechanical coupling efficiency and further reduces myocardial contraction or dilation coordination (26). Noise derived from scars in the raw data is significantly associated with PSD (27). Our results revealed that there was no statistical difference in LVMD parameters between Group 1 and Group 2, which may be due to limited number of patients in Group 1. The multiple stepwise linear regression model also indicated that ischemic extent, diastolic PSD, and NYHA functional classification should be taken into consideration together to predict viable myocardium and myocardial scar, rather than LVMD alone. In addition, the relatively low $R^{2}$ values (0.329 and 0.373$)$ of viable myocardium and myocardial scar indicated that ECG-gated MPI combined with phase analysis and NYHA classification were not completely equivalent to myocardial viability quantification assessed by myocardial perfusion/metabolism imaging.

Previous studies of phase analysis were focused on the application of systolic LVMD in SPECT; little attention was paid to either diastolic LVMD or the application of LVMD in PET, especially in Nitrogen-13 ammonia gated MPI. To date, the pathophysiology and clinical value of diastolic LVMD had not been studied extensively. Similar to systolic LVMD, diastolic LVMD could assess the phase and amplitude of regional myocardial relaxation by multiple Fourier transforms to quantify myocardial discoordination in diastole (23) and appears to provide incremental value in the prediction of adverse outcomes (28). In our study, both systolic and diastolic LVMD were included and it was suggested that diastolic LVMD was 


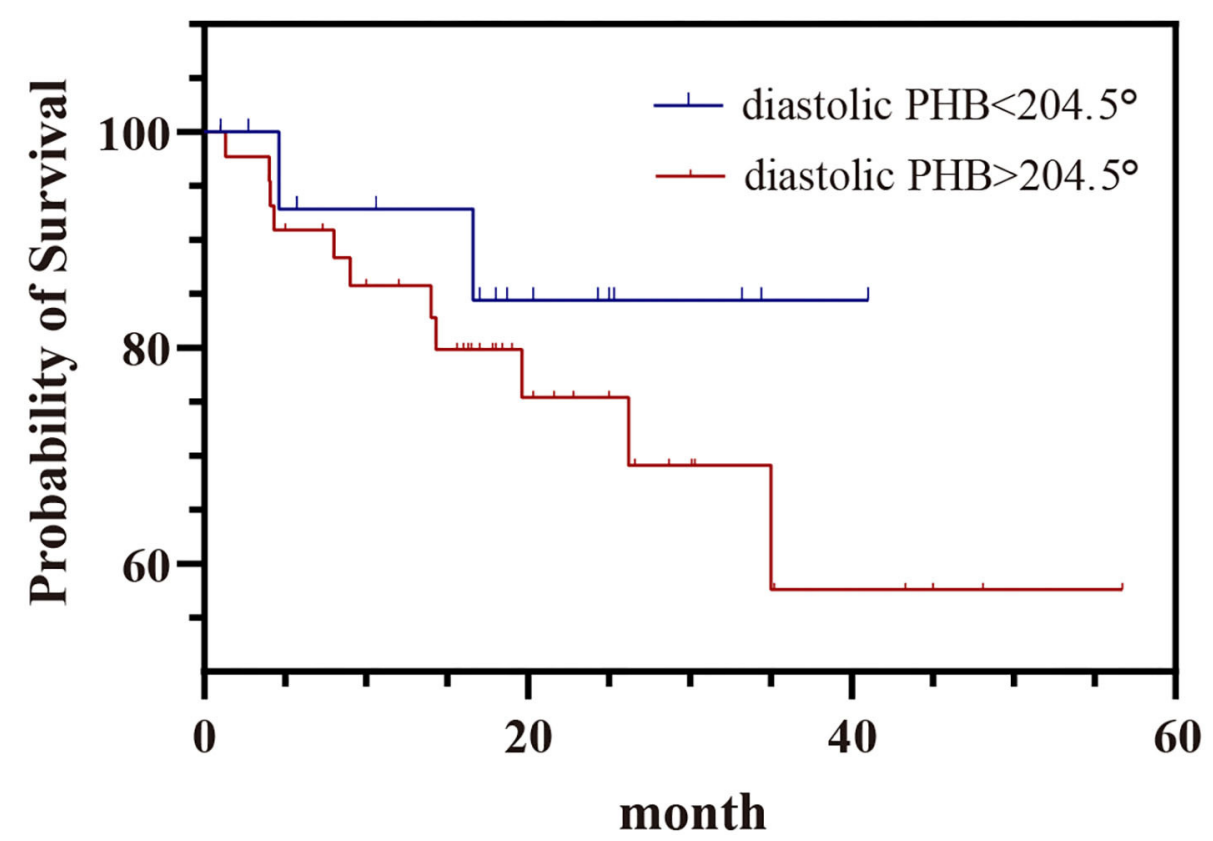

FIGURE 5 | Kaplan-Meier survival curve of MACE in patients with diastolic PHB $<204.5^{\circ}$ and diastolic $\mathrm{PHB}>204.5^{\circ}$. PHB, phase histogram bandwidth.

more strongly associated with myocardial viability, as seen in the diastolic phase histogram. Accordingly, the potential value of diastolic LVMD should be further investigated. Moreover, gPET has the advantage of higher spatial resolution to obtain a more accurate ischemic extent and has the potential to provide myocardial blood flow (MBF) (29). Previous studies suggested that the improvement of LVMD under stress was crucially associated with improved MBF homogeneity (30) and stress MBF was better correlated with LVMD than myocardial flow reserve (31). Thus, the relationship between LVMD and gPET quantitative cardiac functional parameters in Nitrogen-13 ammonia gated MPI is worth further study.

In a cohort of 489 patients with ischemic cardiomyopathy (14), worse LVMD in stress MPI as compared with rest was an independent predictor of all-cause mortality. Systolic and diastolic LVMD were associated with adverse outcomes in CAD patients when the cut-off value was determined by comparison with the normal population (28), and a significantly higher incidence of MACE was also found in patients with systolic LVMD (32). In the present study, although the incidence of MACE in patients with diastolic $\mathrm{PHB}>204.5^{\circ}$ was $25.0 \%$, higher than patients with diastolic $\mathrm{PHB}<204.5^{\circ}(11.8 \%)$, the difference was not significant,which may owe to relatively small samples and short follow-up time. In addition, high proportion of patients with NYHA III and IV (51.9 and $29.1 \%$, respectively) with poor prognosis may interfere the evaluation of the prognostic value of LVMD.

\section{Limitations}

There were several limitations of our study. First, it is a retrospective study with limited number of patients. There were only seven patients in Group 1, which may influence detecting statistically significant differences in LVMD parameters between Group 1 and Group 2. There were reduced number of female patients, which may affect the evaluation of sex. Second, this study was a preliminary attempt to analyze the prognostic value of diastolic LVMD. Larger sample-sized study and longer followup time are needed to further confirm the prognostic value of diastolic LVMD.

\section{CONCLUSION}

LVMD from Nitrogen-13 ammonia gated MPI had added diagnostic value for viable myocardium and myocardial scar. Increased LVMD did not reach a statistically significant predictive value. Further studies are needed.

\section{DATA AVAILABILITY STATEMENT}

The original contributions presented in the study are included in the article/supplementary material, further inquiries can be directed to the corresponding authors.

\section{ETHICS STATEMENT}

The studies involving human participants were reviewed and approved by Human Ethics Committee of Union Hospital, Tongji Medical College, Huazhong University of Science and Technology. The patients/participants provided their written informed consent to participate in this study. 


\section{AUTHOR CONTRIBUTIONS}

DZ conducted the analyses and was a major contributor in writing the manuscript. YL analyzed the images. LZ collected clinical information of all patients. FH and XT acquired GPET images. DJ contributed to revision. WZ provided suggestions about the idea. XL and CQ conceived the idea and contributed

\section{REFERENCES}

1. Garcia MJ, Kwong RY, Scherrer-Crosbie M, Taub CC, Blankstein R, Lima J, et al. State of the art: imaging for myocardial viability: a scientific statement from the American Heart Association. Circ Cardiovasc Imaging. (2020) 13:e000053. doi: 10.1161/HCI.0000000000000053

2. Panza JA, Ellis AM, Al-Khalidi HR, Holly TA, Berman DS, Oh JK, et al. Myocardial viability and long-term outcomes in ischemic cardiomyopathy. $N$ Engl J Med. (2019) 381:739-48. doi: 10.1056/NEJMoa1807365

3. Anagnostopoulos C, Georgakopoulos A, Pianou N, Nekolla SG. Assessment of myocardial perfusion and viability by positron emission tomography. Int J Cardiol. (2013) 167:1737-49. doi: 10.1016/j.ijcard.2012.12.009

4. Dilsizian V, Bacharach SL, Beanlands RS, Bergmann SR, Delbeke D, Dorbala $\mathrm{S}$, et al. ASNC imaging guidelines/SNMMI procedure standard for positron emission tomography (PET) nuclear cardiology procedures. J Nucl Cardiol. (2016) 23:1187-226. doi: 10.1007/s12350-016-0522-3

5. Iiya M, Shimizu M, Fujii H, Suzuki M, Nishizaki M. True complete left bundle branch block reveals dyssynchrony evaluated by semiconductor single-photon emission computed tomography. J Arrhythm. (2018) 35:70-8. doi: $10.1002 /$ joa3.12148

6. Chen J, Garcia EV, Bax JJ, Iskandrian AE, Borges-Neto S, Soman P. SPECT myocardial perfusion imaging for the assessment of left ventricular mechanical dyssynchrony. J Nucl Cardiol. (2011) 18:685-94. doi: 10.1007/s12350-011-9392-x

7. Wang C, Shi J, Ge J, Tang H, He Z, Liu Y, et al. Left ventricular systolic and diastolic dyssynchrony to improve cardiac resynchronization therapy response in heart failure patients with dilated cardiomyopathy. J Nucl Cardiol. (2021) 28:1023-36. doi: 10.1007/s12350-020-02132-1

8. Zhou W, Tao N, Hou X, Wang Y, Folks RD, Cooke DC, et al. Development and validation of an automatic method to detect the latest contracting viable left ventricular segments to assist guide CRT therapy from gated SPECT myocardial perfusion imaging. J Nucl Cardiol. (2018) 25:1948-57. doi: 10.1007/s12350-017-0853-8

9. Karacalioglu AO, Balta S, Emer O, Demirkol S, Celik T, Ozguven M. Phase analysis in patients with reversible perfusion defects and normal coronary arteries at angiography. Ann Nucl Med. (2013) 27:416-22. doi: 10.1007/s12149-013-0700-1

10. Gimelli A, Liga R, Giorgetti A, Favilli B, Pasanisi EM, Marzullo P. Determinants of left ventricular mechanical dyssynchrony in patients submitted to myocardial perfusion imaging: a cardiac CZT study. J Nucl Cardiol. (2016) 23:728-36. doi: 10.1007/s12350-015-0247-8

11. Park S, Cheon GJ, Paeng JC, Won KS, Kang KW, Kim KB, et al. Phase analysis of gated myocardial perfusion single-photon emission computed tomography after coronary artery bypass graft surgery: reflection of late reverse remodeling in patients with patent grafts after coronary artery bypass graft surgery. Nucl Med Commun. (2016) 37:1139-47. doi: 10.1097/MNM.00000000000 00565

12. Hess PL, Shaw LK, Fudim M, Iskandrian AE, Borges-Neto S. The prognostic value of mechanical left ventricular dyssynchrony defined by phase analysis from gated single-photon emission computed tomography myocardial perfusion imaging among patients with coronary heart disease. J Nucl Cardiol. (2017) 24:482-90. doi: 10.1007/s12350-015-0388-9

13. Cho SG, Jabin Z, Park KS, Kim J, Kang SR, Kwon SY, et al. Clinical values of left ventricular mechanical dyssynchrony assessment by gated myocardial perfusion SPECT in patients with acute myocardial infarction and multivessel disease. Eur J Nucl Med Mol Imaging. (2017) 44:259-66. doi: $10.1007 /$ s00259-016-3542-y to analysis and revision. All authors read and approved the final manuscript.

\section{FUNDING}

This work was supported by the National Natural Science Foundation of China (Nos. 81873906 and 81401444).

14. AlJaroudi W, Alraies MC, Menon V, Brunken RC, Cerqueira MD, Jaber WA Predictors and incremental prognostic value of left ventricular mechanical dyssynchrony response during stress-gated positron emission tomography in patients with ischemic cardiomyopathy. J Nucl Cardiol. (2012) 19:958-69. doi: 10.1007/s12350-012-9592-Z

15. Zhang F, Yang W, Wang Y, Tang H, Wang J, Shao X, et al. Is there an association between hibernating myocardium and left ventricular mechanical dyssynchrony in patients with myocardial infarction? Hell J Nucl Med. (2018) 21:28-34. doi: 10.1967/s002449910704

16. Cooke CD, Esteves FP, Chen J, Garcia EV. Left ventricular mechanical synchrony from stress and rest 82Rb PET myocardial perfusion ECG-gated studies: differentiating normal from LBBB patients. J Nucl Cardiol. (2011) 18:1076-85. doi: 10.1007/s12350-011-9455-z

17. Uebleis C, Ulbrich M, Tegtmeyer R, Schuessler F, Haserueck N, Siebermair J, et al. Electrocardiogram-gated 18F-FDG PET/CT hybrid imaging in patients with unsatisfactory response to cardiac resynchronization therapy: initial clinical results. J Nucl Med. (2011) 52:67-71. doi: 10.2967/jnumed.110.078709

18. Juarez-Orozco LE, Monroy-Gonzalez A, Prakken NHJ, Noordzij W, Knuuti J, deKemp RA, et al. Phase analysis of gated PET in the evaluation of mechanical ventricular synchrony: a narrative overview. J Nucl Cardiol. (2019) 26:1904-913. doi: 10.1007/s12350-019-01670-7

19. Patel JJ, Alzahrani T. Myocardial perfusion scan. In: StatPearls. Treasure Island, FL: StatPearls Publishing (2021).

20. Garcia EV, Faber TL, Cooke CD, Folks RD, Chen J, Santana C. The increasing role of quantification in clinical nuclear cardiology: the Emory approach. $J$ Nucl Cardiol. (2007) 14:420-32. doi: 10.1016/j.nuclcard.2007.06.009

21. O'Connor MK, Hammell T, Gibbons RJ. In vitro validation of a simple tomographic technique for estimation of percentage myocardium at risk using methoxyisobutyl isonitrile technetium 99m (sestamibi). Eur J Nucl Med. (1990) 17:69-76. doi: 10.1007/BF00819407

22. Chen J, Garcia EV, Folks RD, Cooke CD, Faber TL, Tauxe EL, et al. Onset of left ventricular mechanical contraction as determined by phase analysis of ECGgated myocardial perfusion SPECT imaging: development of a diagnostic tool for assessment of cardiac mechanical dyssynchrony. J Nucl Cardiol. (2005) 12:687-95. doi: 10.1016/j.nuclcard.2005.06.088

23. Chen J, Kalogeropoulos AP, Verdes L, Butler J, Garcia EV. Left-ventricular systolic and diastolic dyssynchrony as assessed by multi-harmonic phase analysis of gated SPECT myocardial perfusion imaging in patients with endstage renal disease and normal LVEF. J Nucl Cardiol. (2011) 18:299-308. doi: 10.1007/s12350-010-9331-2

24. Sharma RK, Donekal S, Rosen BD, Tattersall MC, Volpe GJ, AmbaleVenkatesh B, et al. Association of subclinical atherosclerosis using carotid intima-media thickness, carotid plaque, and coronary calcium score with left ventricular dyssynchrony: the multi-ethnic Study of Atherosclerosis. Atherosclerosis. (2015) 239:412-8. doi: 10.1016/j.atherosclerosis.2015.01.041

25. Yamamoto A, Takahashi N, Ishikawa M, Abe K, Kobayashi Y, Tamai J, et al. Relationship between left ventricular function and wall motion synchrony in heart failure assessed by ECG-gated myocardial perfusion SPECT. Ann Nucl Med. (2008) 22:751-9. doi: 10.1007/s12149-008-0172-x

26. Nguyên UC, Verzaal NJ, van Nieuwenhoven FA, Vernooy K, Prinzen FW. Pathobiology of cardiac dyssynchrony and resynchronization therapy. Europace. (2018) 20:1898-1909. doi: 10.1093/europace/euy035

27. Ludwig DR, Friehling M, Schelbert EB, Schwartzman D. Impact of scar on SPECT assay of left ventricular contraction dyssynchrony. Eur J Nucl Med Mol Imaging. (2014) 41:529-35. doi: 10.1007/s00259-013-2608-3

28. Fudim M, Fathallah M, Shaw LK, Liu PR, James O, Samad Z, et al. The Prognostic Value of Diastolic and Systolic Mechanical 
Left Ventricular Dyssynchrony Among Patients With Coronary Heart Disease. JACC Cardiovasc Imaging. (2019) $12(7 \mathrm{Pt}$ 1):1215-26. doi: 10.1016/j.jcmg.2018.05.018

29. Camici PG, Rimoldi OE. The clinical value of myocardial blood flow measurement. J Nucl Med. (2009) 50:1076-87. doi: 10.2967/jnumed.108.054478

30. Van Tosh A, Votaw JR, Cooke CD, Reichek N, Palestro CJ, Nichols KJ. Relationships between left ventricular asynchrony and myocardial blood flow. J Nucl Cardiol. (2017) 24:43-52. doi: 10.1007/s12350-015-0270-9

31. Juárez-Orozco LE, Alexanderson E, Dierckx RA, Boersma HH, Hillege JL, Zeebregts CJ, et al. Stress myocardial blood flow correlates with ventricular function and synchrony better than myocardial perfusion reserve: a Nitrogen-13 ammonia PET study. J Nucl Cardiol. (2018) 25:797-806. doi: 10.1007/s12350-016-0669-y

32. Pazhenkottil AP, Buechel RR, Husmann L, Nkoulou RN, Wolfrum M, Ghadri JR, et al. Long-term prognostic value of left ventricular dyssynchrony assessment by phase analysis from myocardial perfusion imaging. Heart. (2011) 97:33-7. doi: 10.1136/hrt.2010.201566
Conflict of Interest: The authors declare that the research was conducted in the absence of any commercial or financial relationships that could be construed as a potential conflict of interest.

Publisher's Note: All claims expressed in this article are solely those of the authors and do not necessarily represent those of their affiliated organizations, or those of the publisher, the editors and the reviewers. Any product that may be evaluated in this article, or claim that may be made by its manufacturer, is not guaranteed or endorsed by the publisher.

Copyright (C) 2021 Zheng, Liu, Zhang, Hu, Tan, Jiang, Zhou, Lan and Qin. This is an open-access article distributed under the terms of the Creative Commons Attribution License (CC BY). The use, distribution or reproduction in other forums is permitted, provided the original author(s) and the copyright owner(s) are credited and that the original publication in this journal is cited, in accordance with accepted academic practice. No use, distribution or reproduction is permitted which does not comply with these terms. 